\title{
UNIVALENT FUNCTIONS AND THE RIEMANN MAPPING THEOREM
}

\author{
P. R. GARABEDIAN ${ }^{1}$
}

\begin{abstract}
A proof of the Riemann mapping theorem is given that depends on variational formulas for univalent functions. The method of proof can be used to simplify the derivation of the ordinary differential equation for extremal univalent functions given by Schiffer in 1938.
\end{abstract}

There are many proofs of the Riemann mapping theorem. Most of them use an extremal problem to establish the existence of the map function. The simplest proofs rely on the theory of normal families of analytic functions to show that there is an extremal function solving the problem considered. Here we present a new proof of this type that is related to the coefficient problem for univalent functions.

The new feature of our proof is a quite elementary variation of univalent functions that is based entirely on the Joukowski transformation. It resembles the method of exterior variation used by Schiffer [2] in the first derivation of his ordinary differential equation for extremal functions arising in the study of the Bieberbach conjecture. The real interest of the new approch lies in the fact that it leads to significant simplifications of that derivation.

Let $\Omega$ be a simply-connected region of the complex $z$-plane possessing at least two boundary points. We may suppose without loss of generality that the point at infinity lies inside $\Omega$. We denote by $S$ the class of univalent functions $g(z)$ defined in $\Omega$ which are normalized so that their Laurent series expansions about infinity have the form

$$
g(z)=z+b_{0}+b_{1} / z+b_{2} / z^{2}+\ldots
$$

Consider the problem of maximizing $\operatorname{Re}\left\{b_{1}\right\}$ within the class $S$. Because $S$ becomes a normal family of analytic functions if a uniform bound is imposed on the translation coefficient $b_{0}$, our extremal problem has a solution $g_{0}(z)$ in $S$. By making appropriate variations we shall establish that the extremal function $w=g_{0}(z)$ maps $\Omega$ conformally onto an infinite region $D$ in the $w$-plane whose boundary $C$ is a horizontal slit. Because the slit domain can be mapped onto the unit circle by an elementary function, this suffices to prove the Riemann mapping theorem.

Received by the editors April 9, 1976 and, in revised form, July 20, 1976.

AMS (MOS) subject classifications (1970). Primary 30A30, 30A34, 30A38.

${ }^{1}$ Work supported by ERDA under Contract E(11-1)-3077 with NYU.

(C) American Mathematical Society 1977 
It is of historical interest that de Possel [1] used our extremal problem to prove the existence of the parallel slit mapping for multiply-connected regions. However, his proof assumed a knowledge of the Riemann mapping theorem, whereas in our approach that theorem becomes the main conclusion.

Our goal is to show that the continuum $C$ bounding the extremal region $D$ in the $w$-plane is a horizontal line segment. Let $A$ and $B$ be any two points of $C$. The inverse Joukowski transformation

$$
\zeta=\frac{1}{2}[w-(A+B) / 2+\sqrt{(w-A)(w-B)}]
$$

maps the exterior of the line segment joining $A$ and $B$ onto the exterior of a circle of radius $|B-A| / 4$. Solving for $w$ in terms of $\zeta$, we see that $\zeta$ is a univalent function of $w$ throughout the exterior $D$ of the continuum $C$. The composition of this transformation with the extremal mapping of $\Omega$ onto $D$ defines a univalent function

$$
\zeta=z+b_{0}-\frac{A+B}{2}+\frac{16 b_{1}-(B-A)^{2}}{16 z}+\ldots
$$

in the class $S$, where $b_{0}, b_{1}, \ldots$ now stand for the coefficients of the extremal function $g_{0}(z)$. Because of the maximum property of $\operatorname{Re}\left\{b_{1}\right\}$, we conclude that $\operatorname{Re}\left\{(B-A)^{2}\right\} \geqslant 0$. This means that the slope of the line segment joining $A$ and $B$ is not greater than 1 in absolute value. Therefore $C$ must be a curve with a nonparametric representation $v=F(u)$ satisfying the Lipschitz condition $\left|F\left(u_{2}\right)-F\left(u_{1}\right)\right| \leqslant\left|u_{2}-u_{1}\right|$.

Denote by $M$ the supremum of the absolute values of the slopes of the chords of the curve $C$. Suppose that $M>0$, and let $\varepsilon$ be any positive number less than $M$. Then there are points $A$ and $B$ on $C$ such that

$$
M-\varepsilon \leqslant|\operatorname{Im}\{B-A\} / \operatorname{Re}\{B-A\}| \leqslant M .
$$

The arc $C_{A B}$ of $C$ between $A$ and $B$ has to lie in a parallelogram with vertices at $A$ and $B$ whose sides have slopes with the absolute value $M$. The diagonal joining $A$ and $B$ has a slope at least as big as $M-\varepsilon$ in absolute value. For small $\varepsilon>0$ this means that the arc $C_{A B}$ is everywhere close to the line segment joining $A$ and $B$.

Now when $R$ is any number in the interval $0<R<1$, we can choose $\varepsilon$ so small that the image of $C_{A B}$ by the inverse Joukowski transformation defined above is a closed curve lying in the circular ring

$$
|B-A| R / 4<|\zeta|<|B-A| / 4 R
$$

of the $\zeta$-plane. Let us apply the Joukowski transformation

$$
w^{*}=\zeta+|B-A|^{2} R^{2} / 16 \zeta,
$$

which maps the domain $|\zeta|>|B-A| R / 4$ onto the exterior of a horizontal slit. By composing the extremal function $g_{0}(z)$ with the transformations from $w$ to $\zeta$ and from $\zeta$ to $w^{*}$, we obtain a comparison function $w^{*}=g^{*}(z)$ of the class $S$ that has the Laurent expansion 


$$
w^{*}=z+b_{0}-\frac{A+B}{2}+\frac{16 b_{1}-(B-A)^{2}+|B-A|^{2} R^{2}}{16 z}+\ldots
$$

about infinity. Since $(B-A)$ is not real, we can take $R$ so close to 1 that

$$
\operatorname{Re}\left\{b_{1}-\frac{(B-A)^{2}}{16}+\frac{|B-A|^{2} R^{2}}{16}\right\}>\operatorname{Re}\left\{b_{1}\right\}
$$

This contradicts the extremal property of $g_{0}(z)$ and shows that the hypothesis $M>0$ was absurd. Thus $C$ is a horizontal line segment, and our proof of the Riemann mapping theorem is complete.

\section{REFERENCES}

1. R. de Possel, Zum Parallelschlitztheorem unendlich-vielfach zusammenhaengender Gebiete, Nachr. Ges. Wiss. Goettingen Math. Phys. Kl. (1) 23 (1931), 199-202.

2. M. Schiffer, A method of variation within the family of simple functions, Proc. London Math. Soc. 44 (1938), 432-449.

Courant Institute of Mathematical Sciences, New York University, New York, New YORK 10012 